\title{
BORED WITH POINT AND CLICK? \\ THEORETICAL PERSPECTIVES ON DESIGNING LEARNING ENVIRONMENTS:
}

\author{
Neville A. Stanton \& Les J. Porter \& Ray Stroud \\ Department of Design \\ Brunel University \\ Runnymede Campus \\ Egham, Surrey, TW20 0JZ, UK
}

\begin{abstract}
Computers have the potential to be exploited as one of the most exciting examples of instructional media. Yet designers often fail to realise this potential. This is, in part, due to the limitations of hardware and software and, in part, due to the lack of good theory developed through conclusive research. Good examples of computer-based learning may owe more to the imaginative flair of the courseware designer, than they do to the application of explicit design guidelines and good learning theory. This paper will therefore consider a variety of issues that may be blocking theoretical development and draw conclusions for future courses of action. This starts with a statement of the problem, first by considering the macro and micro issues, and then by looking at a recent call for help in Computer-Based Learning Environment (CBLE) design. Next, the contribution of instructional design theories will be presented together with a way forward for investigating the issues. Finally the implications for future progress are presented.
\end{abstract}

Keywords: Learning theory, design, instruction, computers

\section{Macro-Issues in design of learning environments}

In a review of the changes in higher education, Newby (1999) identifies a number of themes that are likely to affect the ways in which Universities conduct themselves. The three themes pertinent to this paper are: globalisation of education; widening participation in, and continuation of, education; and developments in information technology. Each of these themes will be considered briefly in turn. Globalisation of education is likely to mean that competition for student populations will no longer be restricted by national boundaries. In commerce, we can see that many corporations compete at a global level, and within Europe the national boundaries are being dissolved with the European parliament. Whilst an international scientific community has always existed for researchers, this has not previously been the case for students. However, it is anticipated that Universities could find themselves competing in a global market for students. The only restricting factor might be the physical mobility of students (especially in the second theme), but the third theme might offer some resolution to this potential problem. In addressing the second theme, we are seeing 
greater participation in education both in terms of continuing professional development and widening access. Governments are particularly keen to promote social inclusion and greater diversity in the student population. Diversity is meant in terms of age, ethnicity, social background and mode of study. This is likely to require greater innovation in teaching methods, both pedagogic and logistic, which will mean considerable investment in teaching resources. The final theme to be considered is that of advances in information technology. As the previous two themes indicate, media that can deliver teaching over national boundaries (coping with delivery over long distances and time zones) to students who may have other life commitments (e.g. work and family) would present a viable solution to higher education in the 21st century. Developments in communications technologies, such as multi-media, communications, video-conferencing and the world-wide web may well have the potential to realise the aims of the international teaching community and greater participation in university education. There are however, some micro issues to be resolved before we are likely to see this happen.

\section{Micro-Issues in design of learning environments}

The micro issues revolve around the delivery of the educational material. If we are to assume that the themes identified by Newby (1999) are going to play a major part in redefining the role of Universities, then we need to consider exactly how the educational technologies will assist. Traditional lecture and tutorial-based teaching will not fulfil this role. Most distance learning course rely largely upon paper-based materials. Computers would seem to offer a unique solution, both in terms of offering multi-media (e.g. film, text, pictures, sounds, etc.) and in terms of a delivery mechanism. Further, with facilities such as email, students can correspond with their tutor and other students. Before this can be done convincingly however, clear instructional guidance for the design of CBLE needs to be developed. A recent enquiry in the Times Higher Educational Supplement (30th April 1999, p.32) makes this point clear. The person making the call for help asked: "My department is using more and more computer-based learning and I detect that some of my students are becoming bored with pointing and clicking. How can this be avoided?" The question received three replies from people engaged in the design of CBLE. The first respondent pointed out that technology is no substitute for inspired content and pedagogical methods. CBLE requires well defined learning objectives and should be based around peerreviewed research material. Further, that students need careful guidance and human contact is essential. The second respondent argued that the enquirer needed to draw upon all of their previous teaching experience when designing the CBLE. It was suggested that CBLE are no different to face-to-face teaching in that an underlying structure was required based on clear outcomes and activities to engage the learner. Finally it was proposed that more success was to be had by getting students working in pairs. The third respondent said that CBLE should be used in conjunction with a core text to enrich learning, primarily because it often lacks the intellectual challenge of a book. However, CBLE can be interesting if it is used appropriately, such as the simulation of experiments as this makes the most of the interactive characteristics. Students will get bored if the tasks are too easy. To summarise these points, the respondents are suggesting that CBLE are likely to be more successful if they: 
- have inspired content and clear objectives

- contain careful guidance and human contact

- draw upon the designer's experience with other technologies

- get students working together

- are used in conjunction with other media

- are used for what they at good at (e.g. interactive simulations)

Despite this advice, which is largely based on previous teaching experience rather than good theory, they are many problems with the design of CBLE. These problems are centred round the design of learning material, design of computer interfaces, and the integration of computers with other media. Unfortunately for the designer, there are many interpretations that they could place on the current guidance and considerable diversity of theoretical perspectives. Added to this, instructional theory has had some spectacular failures in the past (such as all of the research effort that has gone into programmed learning), which is not going to give designers any sense of confidence it contemporary theories.

\section{Developments in design of learning environments}

Developments are only likely to occur through empirical research into instructional theory and a better understanding of how people use CBLE. There are many potential advantages of CBLE including:

- learner pacing of presentation

- active participation in learning

- individualisation

- flexibility

- timeliness and availability

- immediate knowledge of results

- economic factors

(Hudson, 1982; Hobson, 1985; Guest, 1986)

The learner has control over the rate of presentation, which means that differences between learners can be accommodated for. This has a greater advantage over traditional classroom learning by allowing learners to proceed at their own pace. However it has been reported (Dorssett \& Hulvershorn, 1983) that peer learning (two learners working together) reduces learning time further without reducing performance. This is particularly noticeable when there is a mismatch between the ability of the two learners. The majority of people in the peer learning scheme also reported preferring to learn in pairs, rather than alone, showing benefits to be social as well as performance related.

The learners can also have a more active role in their own learning. With other media such as lectures, books and audio visual aids the direction of the communication is mainly one way, from the media to the learner. With CBLE, not only does the medium communicate with the learner, there is also the potential for the learner to with the 
computer. The students' actions can influence what the computer displays next, and so on. Students may find it difficult or embarrassing to stop a lecture, it is certainly impossible to ask a book a question. The CBLE designer is faced with the challenge to make the CBLE interactive, to overcome the boredom associated with point and click. Many implementations are no more interactive than other media (such as books and films) and much less interactive than some lectures.

The degree of interactiveness also influences the individualisation of the learning. It may be very adaptive to the levels of skills, abilities and needs of the individual learner, or present every learner with exactly the same material, in exactly the same sequence regardless of the specific needs of the learner. Further the medium can ensure that mastery of one topic is complete before the learner is allowed to progress to the next (Patrick et al, 1986), ensuring that learners do not get out of their depth. This may also help to prevent any of the learners getting to a point where they are unable to proceed further.

CBLE is flexible along two major dimensions. The hardware may be used for the learning of individuals and as a tool for many other tasks (such as word processing, drawing, accounting, storage of records etc.). The software may be adapted for learning many different tasks. This may include minor alterations when one of the tasks changes slightly, or when an aspect of the learning is found to impair transfer. The inherent flexibility of the computing medium is that it can be adapted for many different tasks.

As a learning medium the computer has the advantages associated with more personal learning media (such as printed materials) including those of timeliness, availability, localisation and even portability (particularly with the increasing power and sophistication accompanying lap-top micro computers). These developments make CBLE an even more attractive medium. Learners are able to undertake learning at a time and in a place that is convenient to them. From the point of view of the tutor, CBLE can have distinct advantages over traditional media (Guest, 1986) including: allowing the learner active participation in learning, self-pacing of instruction, and immediate knowledge of. Related to the individualised and interactive aspects of CBLE is the role and nature of feedback in learning. There is the potential for feedback to be very close in proximity to the learners' actions. The short time loop between action and knowledge of results can be exploited to the full through interactive learning environments.

Economic factors are also an important consideration in the choice of instructional medium. A variety of factors may lead to CBLE fulfilling this criteria for selection. These include the issues of flexibility of the hardware and software already raised. Also the courseware is reusable. CBLE may reduce the time it takes the learner to become proficient, and it can make expert knowledge more widely available (Lewis \& Mace, 1988). CBLE can be used in more than one place at the same time. This could also be true for lectures if closed circuit television was used, but then interactivity is lost. Once the capital equipment and authoring software is purchased, the ongoing costs are limited to the authors' time. The author can remain remote from the end users. O'Neil 
\& Paris (1981) cite the advantages of CBLE as those of predominately reducing cost and increasing effectiveness.

\section{Instructional Design Theory}

Ironically, Annett and Sleeman (1971) reported that CBLE was being held up by technology rather than learning theory, whereas nearly three decades later the technology has kept its' promise and the learning theory has proved inadequate. Most notably the failure of programmed instruction has led to the realisation that learning is more complex process than originally considered in behaviourist research. The approach seemed to be grounded in good theory, by adapting Skinnerian principles to linear teaching machines, pre-dating desktop computing technology. The learning theory was based upon operant conditioning, where a stimulus (e.g. a question) was followed by a response (e.g. the students answer), which was reinforced with a reward (e.g. a credit for getting the answer correct). This process of learning had been well researched in animal learning, and shown to produce a wide range of behaviour outside the animals normal repertoire. Programmed learning was simply applying the same principles to human learning. The approach was ordered, using pre-defined instructional sequences and small, simple, learning steps to avoid erroneous responses. Student proceed at their own pace, and the correct answer given after each response. All told, the theory would seem to hold great promise. Unfortunately however, the evaluation data was equivocal. There was no conclusive proof that it worked and, subsequently after two decades of research, the theory has been abandoned in instructional design.

By the early 1970's it was becoming clear that teaching was an intelligent process, and that traditional programmed learning was too inflexible. A cognitive approach to the understanding of learning was being presented. In marked contrast to programmed learning it was considered that learning should be a more interactive process, enabling learners to see the consequences of their actions, and further providing them with the means of correcting inadequate solutions. The change of emphasis was on allowing the learner greater freedom within the problem space to not only achieve the correct solution, but also to get things wrong, and put them right again. In the early 1980's the availability of low budget computers has revived an interest in machines that can teach.

Effective instructional design should be influenced by an extensive understanding of the processes involved in the acquisition of skills and knowledge. Unfortunately this is not the case, as Norman (1980) observed, this key issue (learning) still largely remains elusive. Learning appears to involve:

"continual exposure to the topic, probably accompanied by several bouts of restructuring of the underlying mental representations, reconceptualisations of the concepts, plus many hours of accumulation of large quantities of facts." (Norman, 1980)

Learning is a complicated and time consuming process, and it is difficult to study. However, there is an extensive range of instructional theories for the designer to choose from. They each tackle the problem in a different way. In a review of theories, 
Stammers \& Morrisroe (1985) showed that they differ on several dimensions. First there is the concern for creating a mental set, so that incoming information can be absorbed in the right context. Next is the division and subdivision of the material, either to be built up or filled in by the learner, depending on the theoretical perspective advocated, e.g., a top down, bottom up, or filling in mode. Another dimension was to consider the content of the material to be learnt, and at which cognitive level it applied. A final dimension covers the level of individualism in the learning process, with material structured to best suit the individual's own particular cognitive style. Whether this is done intelligently by the system, is preset on the basis of pretesting, or is structured by the individual themselves is another issue. It is worth considering a number of classic learning theories, (e.g., assimilation to schema; elaboration; web learning; levels of learning; and multi-level) which are media independent. These are presented in brief below.

The assimilation to schema theory (Ausubel, 1969) suggests that before effective learning can occur, it is first necessary for the learner to possess the correct schema through which to receive the incoming information. Once this is in place, the learner is then able to build on existing knowledge in the right context. This theory can be linked to Niesser's (1976) notion of the schema as an interpreting mechanism that makes sense of incoming information. An incorrect contextual set may increase the possibility of misinterpretation, and therefore reduce the effectiveness of learning.

The elaboration theory suggests that instruction should begin with the general outline of the topics to be covered. This, in effect, orientates the learner as to what to expect in the instruction to follow. The elaboration then given divides and subdivides the material into parts to yield more detail until the desired level of learning is reached. Reigeluth (1983) described elaboration theory using the 'zoom lens' analogy. the learner starts with the wider angle view (major parts and major relationships), and then zooms in one level on a given part to see more about each of the major subparts. Having studied those subparts and their interrelationships the learner can them zoom out to the wide angle view to review the subparts within the whole picture. Reigeluth suggested that there may be a restriction placed on learners that prevents them from viewing anything that they have not viewed from a higher level.

The web learning theory (Norman, 1982) suggests that instruction takes the form of an initial outline or supporting web structure. This is then progressively built up as more and more details are filled in as learners build up appropriate cognitive structures. This theory shows some similarity to assimilation to schema theory and elaboration theory by providing overview information before presenting detail. Many of the theories overlap conceptually, which is not too surprising as they are describing the same phenomenon, i.e. the learning process. Perhaps the highest gain is to be had with some blend or amalgamated theory, which combines the best elements of all the perspectives.

The levels of learning theory suggests that instruction should proceed from the lower levels of learning up to the higher levels. Movement up to the next level only occurs 
when understanding is complete at each level. Gagné (1977) who classified levels of learning by increases in complexity. The levels were:

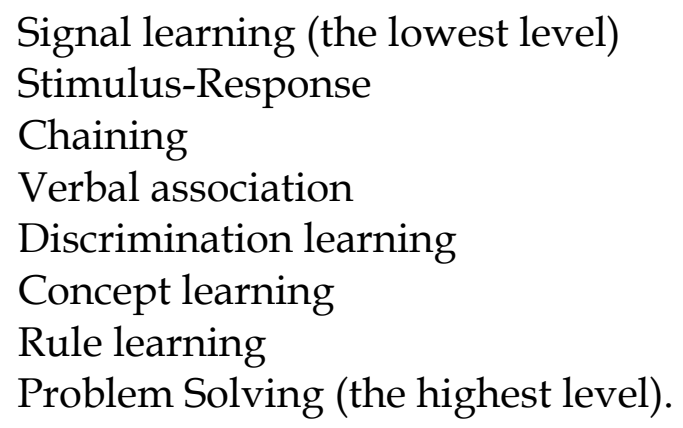

Gagné further proposed four stages of a learning sequence: apprehension, acquisition, storage and retrieval. A more recent level of learning theory has been proposed by Merrill (1983) called 'component display theory'. In this theory Merrill classifies learning objectives along two dimensions, type of content and level of performance. Under content his levels are: fact, concept, procedure and principle. His stages, or levels of performance, are: remember, use and find.

The multi-level theory suggests that learning occurs at many levels, and this will depend upon the learner's own individual style. Some individuals will be able to tolerate uncertainty in learning and others will not. This tolerance may make certain learners more suited to top-down instruction and other more suited to bottom-up instruction. Pask (1976) suggested two broad ways in which learners may differ in their approach to learning. He called these 'holist' and 'serialist' learners. His proposal suggests that individual differences in learning style are worthy of further investigation. Holists tackled several aspects of a topic at the same time, adopting a global, thematic, approach involving the development of broad descriptions. Serialists concentrated on detail and procedure, conceptualising information in a linear structure. Other theorists have conceived of other dimensions such as field dependence (Witkin et al, 1977), creativity (Kirton, 1994) and problem solving styles (Kaufman, 1989). A good review of learning styles may be found in Riding \& Raynor (1998). The multi-level theory is different from the first four in its attention to the individual at the micro-level. Whereas the other theories operate at the macro-level in attempting to provide an overall instructional design theory.

Clearly there are many factors that effect the transfer of instruction, but these will certainly include the following:

- practice and repetition

- feedback and knowledge of results

- task difficulty

- psychological orientation

- intelligence

- motivation

- experience 
(Stammers, 1985, b; Mahoney \& Lyday, 1984; Sklaver, 1986;

Morris \& Rouse, 1985; Pintrich, Cross, Kozma \& McKeachie, 1986;

Keller, 1983)

The failure of theorists to produce a unified, fully validated, instructional theory can be understood more clearly when the number of possible variables are identified. This is because such a theory would have to allow for such variables as e.g.: factors contributing to instruction and learning, the task to be learnt and individual characteristics of the learner. For example, four dimensions of motivation have been identified (Keller, 1983) which are: student interest, relevance of material, student expectancy and satisfaction. For each of the dimensions, Keller puts forward strategies to be incorporated in instructional design. These can be regarded as a cookbook formula rather than relying upon any theoretical underpinning. The interest dimension refers to the arousal of the learners' curiosity, and the extent to which this is sustained over time. Relevance is the degree to which the learners perceive the instruction to help achieve their goals. The expectancy dimension relates to the learners' perception of their likelihood of success and the extent to which this is seen to be under their control. Satisfaction is regarded as the learners' intrinsic motivations and their reactions to extrinsic rewards. The motivational aspect of learning is regarded as one of the most important learner characteristics, which together with intelligence, influences the success of a programme (Pintrich, Cross, Kozma \& McKeachie, 1986).

In summarising the theoretical perspectives, three main themes emerge. First, the situation and context of the learning is important in helping the learner assimilate the information. These themes emerge from the instructional theories emphasising the importance of orientating the learner to the material (i.e. assimilation-to-schema, elaboration theory, and web learning theory). Second, the structuring and classification of the material can help determine the sequence of instruction, such as classification in terms of levels of complexity. These themes emerge from the instructional theories emphasising the importance of dividing the learning material into manageable chunks (i.e. levels-of-learning and component display theory). Third, the contradictory notions of individualisation of learning are contrasted against learning as a social process. These themes emerge from the instructional theories emphasising the importance considering differences between learners as well as learners learning in groups (i.e. multi-level theory and the ideas surrounding peer learning). It was noted in a survey of designers of CBLE that the design process was the most difficult, and yet most important, part of the procedure (Learning Technology Unit, 1988). Task analysis was one of the tools that designers use for the structuring of data about the task. This technique also lends itself as a natural structure for the learning modules, and has been put forward by Shepherd (1985) as a tool for facilitating learning design. Shepherd claimed that it not only serves to clarify the content of what should be learnt, but it also clarifies the learning objectives. The use of task analysis enables a hybrid approach to instructional design. It is possible to incorporate elements of elaboration, a webbed network, assimilation and a multi-level approach. In addition the content of the material needs some formal analysis, and content analysis is one such technique (Stanton \& Baber, 1996). 


\section{Content analysis of learning material for CBLE}

Content analysis (Kirakowski \& Corbett, 1989; Robson, 1993) is a technique that has been around since the beginning of the century for analysing the content of documents. The term 'documents' refers to all media: newspapers, diaries, speeches, letters, reports, books, journals, notices, films, photographs, videos, radio and television programmes. Stanton \& Baber (1996) propose using content analysis for CBLE design in 5 main stages as shown in figure 1.

\section{FICURE ONE ABOUT HERE}

\section{Figure 1. Content Analysis Process.}

\section{Determine objectives}

The starting point of the process is to determine the objectives for the use of the material, for example the material may be intended for use as an encyclopaedia, teaching material, learning materials, a journal, support a help desk, a database etc. Each of these different objectives may engage the user in different types of activity and therefore require the content to be structured in a different manner. Some material may be used for more than one type of activity and therefore require structuring in more than one way. CBLE has a distinct advantage over traditional media by virtue of enabling different structures to be placed upon it.

\section{Define unit of analysis}

Based upon the objectives and the use to which the material is to be put, the next task is to define the unit of analysis. The unit could be, for text; individual words, phrases, sentences, paragraphs, etc. For pictures the unit of analysis could be an object, frame, sequence of frames, etc. It is also necessary to consider the context within which the unit occurs, as this will affect the meaning of the unit. Software tools exist for identifying keywords, keywords in context and combined criteria lists. Such tools make the task of analysing large documents much easier.

\section{Construct categories for analysis}

The construction of categories will be based upon the considerations above (objectives and unit of analysis). Generic categories might be formed from subject matter, objects, authors, countries, etc. It is desirable that the categories are exhaustive and mutually exclusive. This ensures that everything can be categorised and reduces the ambiguity of categorisation. The analysis will be no better, or worse, than the system of categorisation.

\section{Test coding to assess reliability}

To determine the objectivity of the coding and categorisation system, it is necessary that the scheme be tested. Typically a sample of the material is taken and coded by at least two persons who have been trained in using the coding and categorisation system by the person who devised it. The categorisations made by these persons should be tested for reliability. If reliability is low (i.e. there is little agreement on which units should be 
assigned to the categories) then the coding scheme may need revising or more training in the coding scheme may be required. If reliability is high (i.e. there is a high degree of agreement on which units should be assigned to the categories) then the scheme is ready to be used.

\section{Conduct analysis}

The content of the documents can be analysed according to the devised scheme. The analysis is continually open to verification and checks of reliability. Indeed content analysis should be considered as a continual process, as the feedback arrows in figure 1 indicate. The analyst can change the objective, unit of analysis and construct new categories, but each change should undergo some form of testing. The advantages of using content analysis to assist in the design of CBLE systems are that it can be used on existing documents, it enables re-analysis for reliability checks and it relatively cost efficient compared with designing a CBLE system from scratch. However, one must bear in mind that bias or distortion could be introduced into the analysis and that the documents were originally produced for another purpose.

\section{Conclusions}

To conclude, this paper has attempted to indicate why difficulties have been encountered in the design, and use, of CBLE. The research evidence suggests that the present status of knowledge is at best somewhat wanting and at worst contradictory and unhelpful. To improve this state of affairs requires thorough investigation of theoretical approaches (both pure and mixed), learning environments (both pure media and mixed media), different types of learning task, different subject domains, and individual differences in students. Studies tend to concentrate on a sub-set of these issues, which means that progress is painfully slow. The three main themes that emerge from the analysis of instructional design theory are the importance of situation and context, structuring and classification, individualisation It is difficult to predict the future, but it would be gratifying to think that outcomes of the research would lead to:

- convergence of theoretical perspectives

- adaptation of theories to CBLE design

- clearer CBLE design guidance

- integration of CBLE into holistic learning environment

The pessimistic view of the research effort is that it will continue in a muddled fashion and the theories would fail to inform CBLE design. Certainly equivocal evaluations of CBLE would lead to the abandonment of research and development in this area, in the same way as programmed learning was downcast. Continuation of the point-and-click approach to design may well be symptomatic of poor instructional design theory. The optimistic view is however, that we will see learning gains with CBLE which will lead to insight into good design principles and the further understanding of learning and development of good, useful, learning theory.

\section{Acknowledgement}


Parts of this paper were presented by the first author in an invited address at Challenges ' 99 held at the University of Minho, Braga, Portugal. The first author is grateful to the organisers and the questioners for comments he received which have sharpened some of the ideas presented in this paper. The authors are grateful to the reviewers and editors of this journal for their help in shaping this paper.

\section{References}

Annett, J. \& Sleeman, D. H. (1971) Report on the current state of research and development of computer-assisted instruction (and related projects) in the U.S.A. Unpublished manuscript.

Ausbell (1968) Educational psychology: A cognitive view. Holt, Rienhart \& Winston: New York.

Dorssett, D. L. \& Hulvershorn, P. (1983) Increasing technical training efficiency: Peer training via computer-assisted instruction. Journal of Applied Psychology 64 (4) 552-558

Gagne , R. M. (1985) The conditions of learning and theory of instruction. CBS College Publishing: New York.

Hobson, P. G. (1985) Microcomputers in technical training: the MicroTICCIT system. Telecommunications for Pacific Development PTC '85, Toward a Digital World, Honolulu, U.S.A. 380-383.

Hudson, W. J. (1982) Computer-based training: Will it replace you? CTM: The Human Element 15, 13-21.

Kaufmann, G. (1989) The assimilator-explorer inventory. Cited by: Riding, R. \& Raynor, S. (1998) Cognitive Styles and Learning Strategies. Fulton: London.

Keller, J. M. (1983) Motivational design of instruction. In: Reigeluth, C. M. (editor) Instructional Design Theories and Models: An Overview of Their Current Status. LEA: New Jersey, 383-434.

Kirton, M. J. (1994) Adaptors and Innovators. (second edition) Routledge: London.

Kirakowski, J. \& Corbett, M. (1989) Effective Methodology for the Study of HCI. Amsterdam: North-Holland.

Learning Technology Unit (1988) Authoring of Computer Based Training Materials ESRC: London. 
Lewis, R. And Mace, T.D. (1988) Support tools for Authoring - a seminar report. Occasional Paper ESRC-InTER/7/88, Department of Psychology: Lancaster.

Mahoney, F. X. \& Lyday, N. L. (1984) Design is what counts in computerbased training. Training \& Development Journal, 38, 40-41.

Merrill, M. D. (1983) Component display theory. In: Reigeluth, C. M. (editor) Instructional Design Theories and Models: An Overview of Their Current Status. LEA: New Jersey, pp. 279-333.

Morris, N. M. \& Rouse, W. B. (1985) The effects of type of knowledge upon human problem solving in a process control task. IEEE Transactions on Systems, Man. And Cybernetics. 15 (6), 698-707.

Newby, H. (1999) Higher Education in the twenty-first century. University of Southampton, Southampton.

Niesser, U. (1976) Cognition and Reality. Freeman: San Francisco.

Norman, D. (1980) Twelve issues for cognitive science. Cognitive Science 4, $1-32$.

Norman, D. A. (1982). Learning and memory. San Francisco: Freeman.

O'Neil, H. F. \& Paris, J. (1981) Introduction and overview of computer-based instruction. In: O'Neil, H.F. (editor) Computer-Based Instruction: A State-ofthe-Art Assessment. Academic Press: New York.

Patrick, J. (1992) Training: Research and Practice. Academic Press: London.

Pask, G. (1976) Strategies and styles of learning. British Journal of Educational Psychology, 46, 128-148.

Pintrich, P.R., Cross, D.R., Kozma, R. B. and McKeachie, W.J. (1986) Instructional Psychology. Annual Review of Psychology 37, 611-651.

Reigeluth, C.M. \& Stein, F.S. (1983) The elaboration theory of instruction. In: Reigeluth, C.M. (editor) Instructional Design Theories and Models: An Overview of Their Current Status. LEA: New Jersey, 335-381.

Riding, R. \& Raynor, S. (1998) Cognitive Styles and Learning Strategies. Fulton: London

Robson, C. (1993) Real World Research. Oxford: Blackwell. 
Shepherd, A. (1985) Hierarchical task analysis and training decisions. Programmed Learning \& Educational Technology. 22 (2), 162-176.

Sklaver, E. R. (1986) Dynamic simulation in 1991: an Exxon viewpoint. Proceedings of the Computer Simulation Conference. Reno, NV, U.S.A, 292296.

Stammers, R. B. (1985) Instructional psychology and the design of training simulators. In: Walton, D. G. (editor) Simulation for Nuclear Reactor Technology. University Press: Cambridge, 161-176.

Stammers, R. B. \& Morrisroe, G. C. (1985) Operator Training in Future OnBoard Naval Command Systems. MOD Contract No. 2097/047 AMTE(T) Aston University: Birmingham.

Stanton, N. A. \& Baber, C. (1996) Content Analysis: anew approach for authors and users of hypermedia. In: Jones, H. \& Earnshaw, R (Eds) Digital Media and Electronic Publishing. Academic Press: London.

Whiteside, T. C. D. (1983) Simulators and realism. Quarterly Journal of Experimental Psychology 35A, 3-15.

Witkin, H. A., Oltman, P.K., Raskin, E. \& Karp, S. A. (1971) A Manual for the Embedded Figures Tests. Consulting Psychologists Press: USA. 


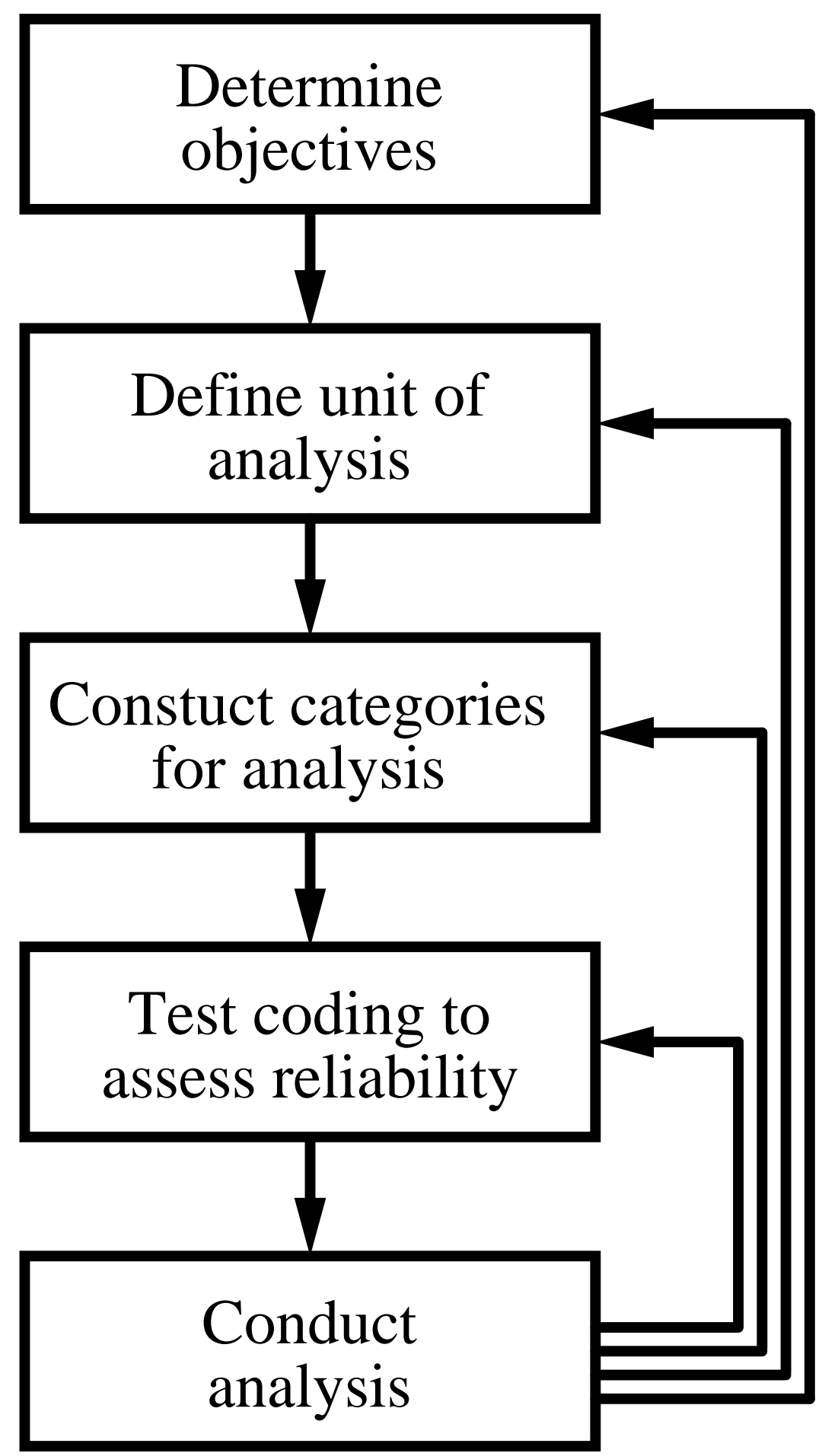

FIGURE ONE 